\title{
Biochemical Changes Induced by Embryonic Substantia Nigra Transplanted to the Striatum of Parkinsonian Monkeys
}

\author{
J.D. Elsworth, J.R. Sladek Jr. ${ }^{1}$, D.E. Redmond Jr., J.R. Taylor, T.J. Collier ${ }^{2}$ and R.H. Roth \\ Departments of Pharmacology and Psychiatry, Yale University School of Medicine, New Haven, CT 06510, \\ ${ }^{1}$ Neuroscience Institute, The Chicago Medical School, Chicago IL 60064 and ${ }^{2}$ Department of Neurobiology \\ and Anatomy, University of Rochester School of Medicine, Rochester, NY 14642, USA
}

Parkinsonian behavioral deficits are improved following transplantation of fetal dopamine (DA) neurons to the striatum of MPTP-treated monkeys, although the mechanism of the recovery has not been resolved. While there is good evidence for the survival of grafted tyrosine hydroxylase (TH)-positive cells following their placement in caudate nucleus, it is not known whether they provide sufficient DA to the caudate nucleus to account for the behavioral effects of transplantation.

Adult male vervet monkeys, which had exhibited stable parkinsonism for one year after systemic MPTP $\mathrm{HCl}$ administration $(2 \mathrm{mg} / \mathrm{kg}$ i.m. over 5 days) were the hosts. Three of these animals received solid allografts of vervet embryonic substantia nigra stereotactically implanted bilaterally at several sites in the caudate nucleus. One monkey was grafted with the mesencephalon from a 44 day old embryo (age post-conception, assessed by ultrasound measurements). The mesencephalon from a 49 day old embryo was divided between two other monkeys. Gestation is 165 days in this species. Following sacrifice three months later, the brain of each monkey was perfused with chilled saline, removed and tissue punched bilaterally from chilled coronal sections. Tissue punches were frozen in liquid nitrogen until assayed for concentrations of DA, homovanillic acid (HVA), determined by HPLC and GC-MS respectively. The remaining sections of brain were post-fixed and stained for the presence of $\mathrm{TH}$.

DA concentration in central and medial substantia nigra of the host monkeys was depleted more than $90 \%$ compared to untreated controls $(n=10-13)$. This magnitude of depletion in the substantia nigra was at least as great as that found in the substantia nigra of severely parkinsonian MPTP-treated monkeys that were not transplanted $(n=11)$. Thus, the severity of the MPTP lesion in the host monkeys was confirmed. Large numbers of well-defined THpositive fibers were observed in the caudate nucleus of the grafted animals. DA concentrations were raised by up to $50 \%$ of untreated control levels in tissue sampled near to the grafts compared to that distant from the grafts (Fig. 1A). At sites proximal to grafts HVA/DA ratios were normalized (Fig. 1B). At positions distal to the grafts, DA levels and HVA/DA ratios were not different from those measured in MPTP-treated monkeys without grafts $/ 1 /$.

DA levels near to the grafts were well within the range observed in asymptomatic MPTPtreated vervet monkeys (greater than $5 \%$ of control). The normalized HVA/DA ratios may reflect synthesis and release of DA by transplanted cells in the absence of external host to graft neuronal connections, or may indicate autoregulation of metabolic activity in the grafted cells.

These data are consistent with the hypothesis that transplanted embryonic mesencephalic tissue can provide sufficient DA for restoration of the biochemical balance of the dopaminedepleted basal ganglia in primates.

Supported by NS-24032, Axion Research Foundation, St. Kitts Biomedical Research Foundation

\section{REFERENCE}

1. Elsworth JD et al. Neuroscience 1989; 33: 321-333. 


\section{A. Elevated DA level}

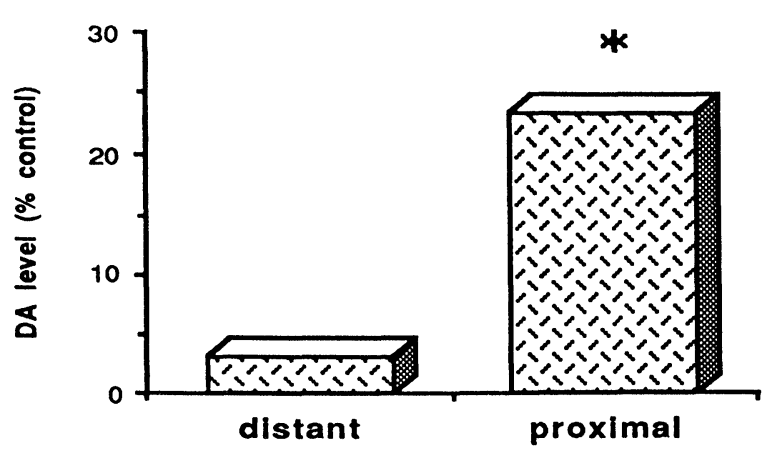

B. Normalized HVA/DA ratio

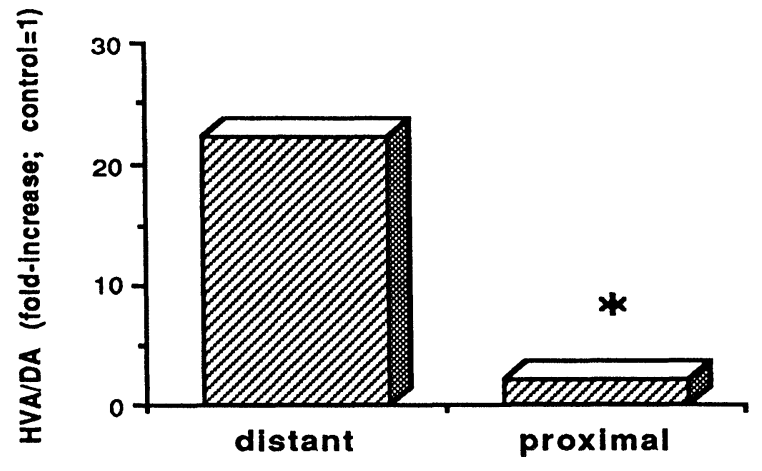

DA levels and HVA/DA ratios in ventromedial caudate nucleus of 3 transplanted MPTP-treated monkeys, compared to non-MPTP-treated controls. Proximal, data from near a graft; Distal, data from tissue not in the vicinity of a graft. 

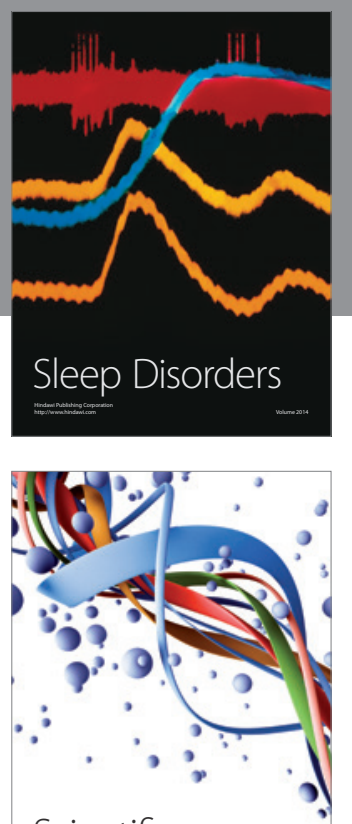

Scientifica
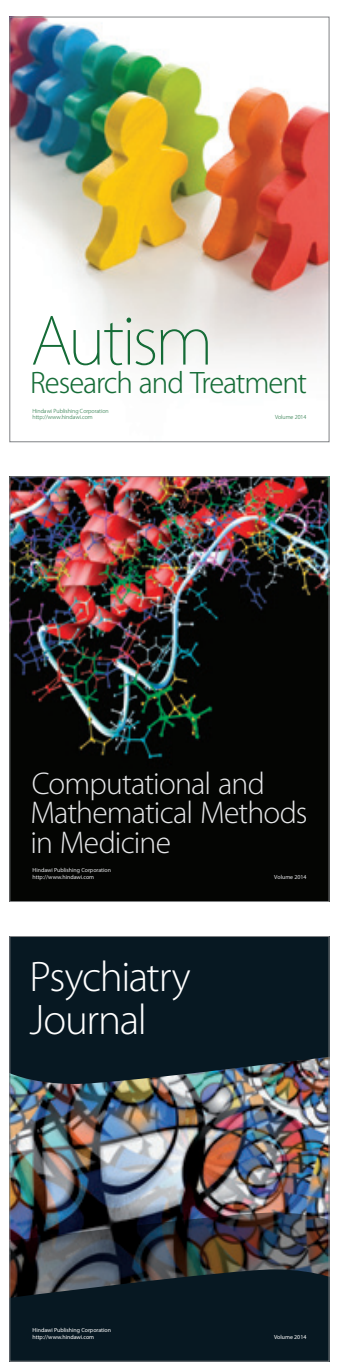
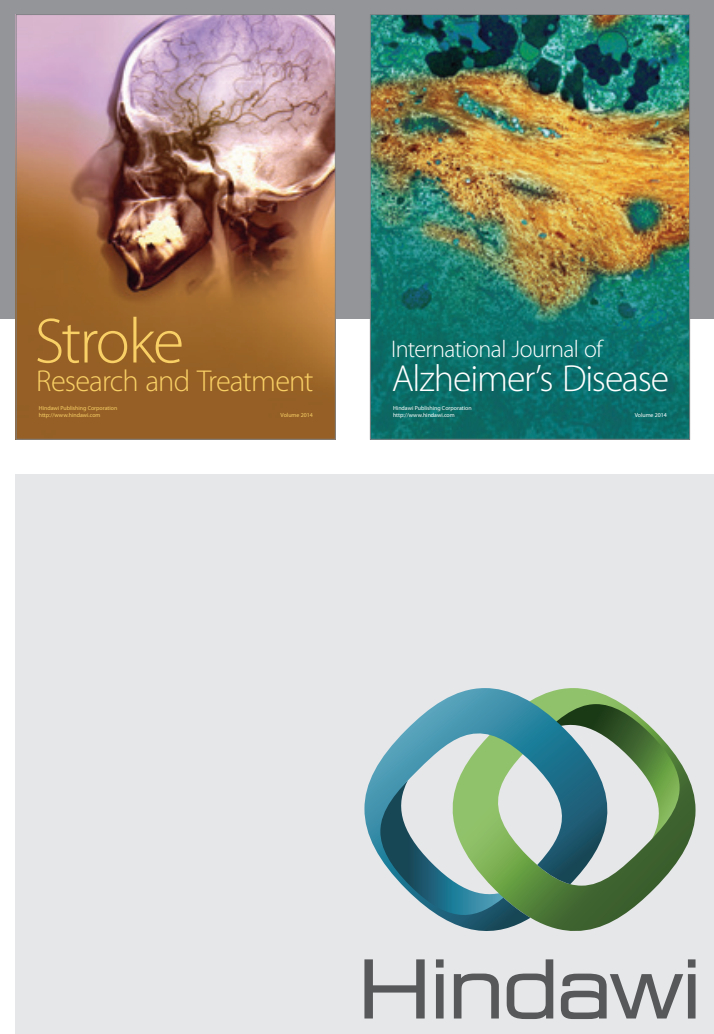

Submit your manuscripts at

http://www.hindawi.com
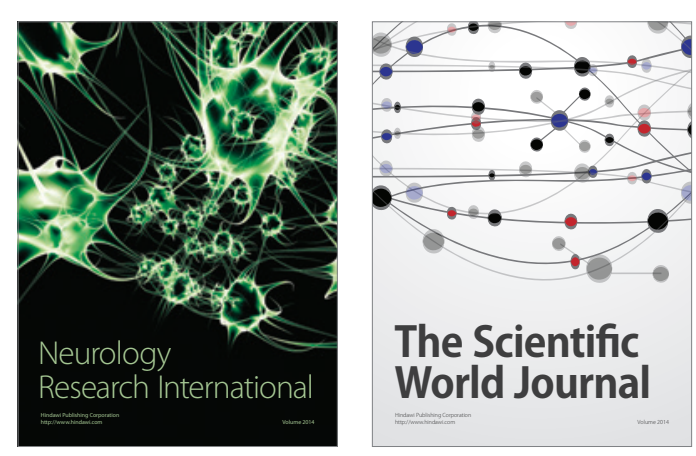

The Scientific World Journal

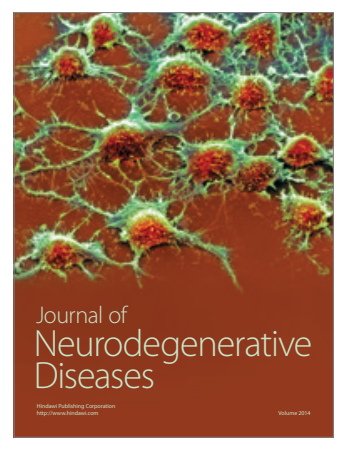

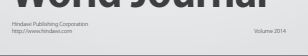

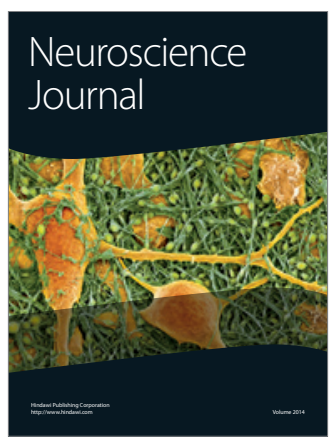

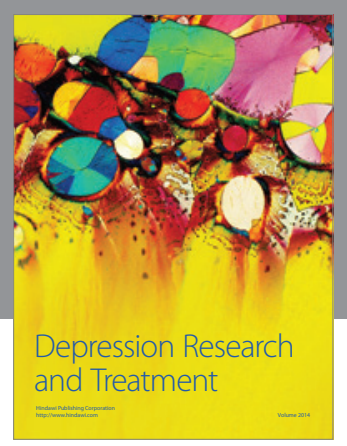
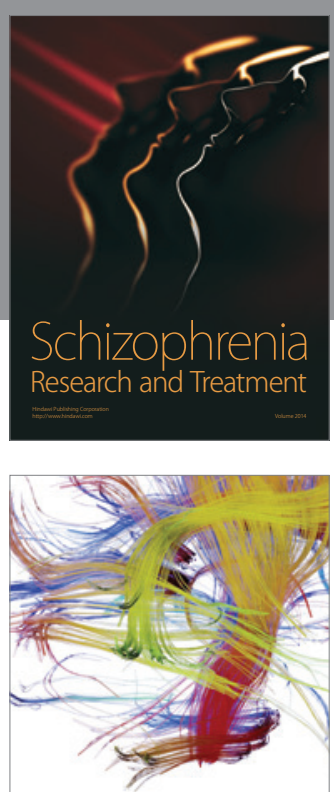

Brain Science

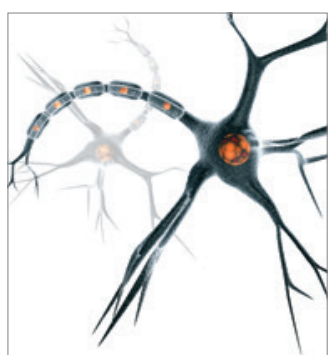

Neural Plasticity
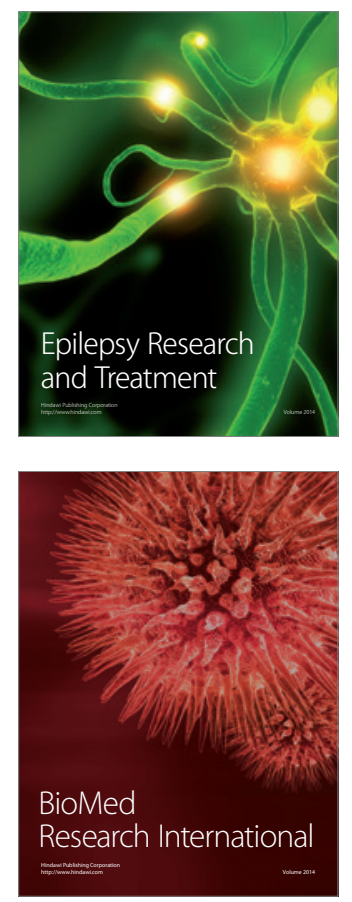

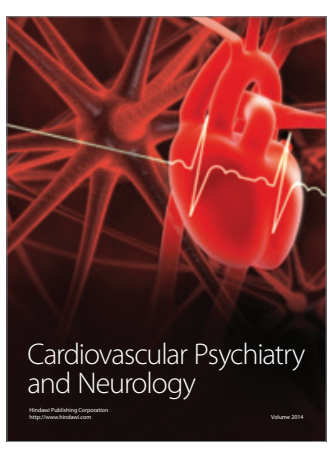

Parkinson's

Disease
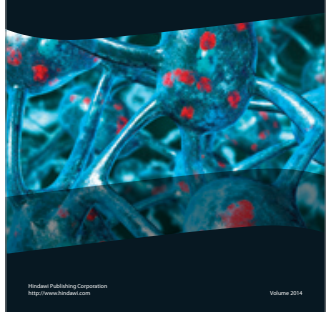